\section{Machine dilemma}

Igor Aleksander

The Age of Intelligent Machines. By Raymond Kurzweil. MIT Press: 1990. Pp. 565. £19.95 (£29.95 after 31 March 1991), \$29.95 (\$39.95 after 31 December 1990).

FourteEn-year-old Margaret Litvin, in her seventh-grade essay on "living in the age of intelligent machines", has written

All right, suppose that we have an artificial brain that is in all senses equivalent to ours... Why should anyone be attracted to this repulsive idea?

Besides, I think most reasonable people would agree with me that it won't work anyway." Raymond Kurzweil has included this extract in his collection of the thoughts of those working in artificial intelligence (AI) who evidently have been attracted to the repulsive idea. Unwittingly, he has pointed to a dilemma that sits squarely on the shoulders of anyone working in the field that his book celebrates. Artificial intelligence is now more than 40 years old (although roots may be traced further back). It is concerned with doing things on computers that, if done by humans, would be thought to require intelligence. A massive amount of scientific, intellectual and financial investment appears to have resulted in very little, either as a pointer to the way our brains work (classical AI researchers are quite adamant about not being interested in how the brain works - only in apeing what it does) or improvements in the usefulness of computers. So the dilemma lies in the fact that while doing research in $\mathrm{Al}$ may be enormously stimulating for those doing it, justification for it and predictions of what it would achieve have mostly been ill-founded.

Kurzweil is living proof of the nature of this dislocation. He is hailed as one of the leading inventors in the United States for having produced a reading machine that scans written text and turns it into synthesized speech. This is of considerable benefit to the blind, and may be found doing useful work in many libraries and schools. But, such a useful machine has been produced with virtually no appeal to the work of AI researchers - it is based on a high degree of engineering skill, exploitation of what computers do well anyway, and Kurzweil's unbounded entrepreneurship. If machines have proved to be useful for a large number of people it is in the areas of spreadsheets, word processors and databases and not in trying to be like humans.

As Kurzweil's invention of the reading machine was a heroic act, so is his book, The Age of Intelligent Machines. It is large, heavy, contains a mixture of contributed and self-authored passages, photographs of self-satisfied looking AI geniuses, and sports a mixture of writing styles ranging from short essays to platonic dialogues with highly illustrated explanatory passages somewhere in between. Virtually the whole of civilized thought is seen as the basis of artificial intelligence: in the first part of the book the reader is

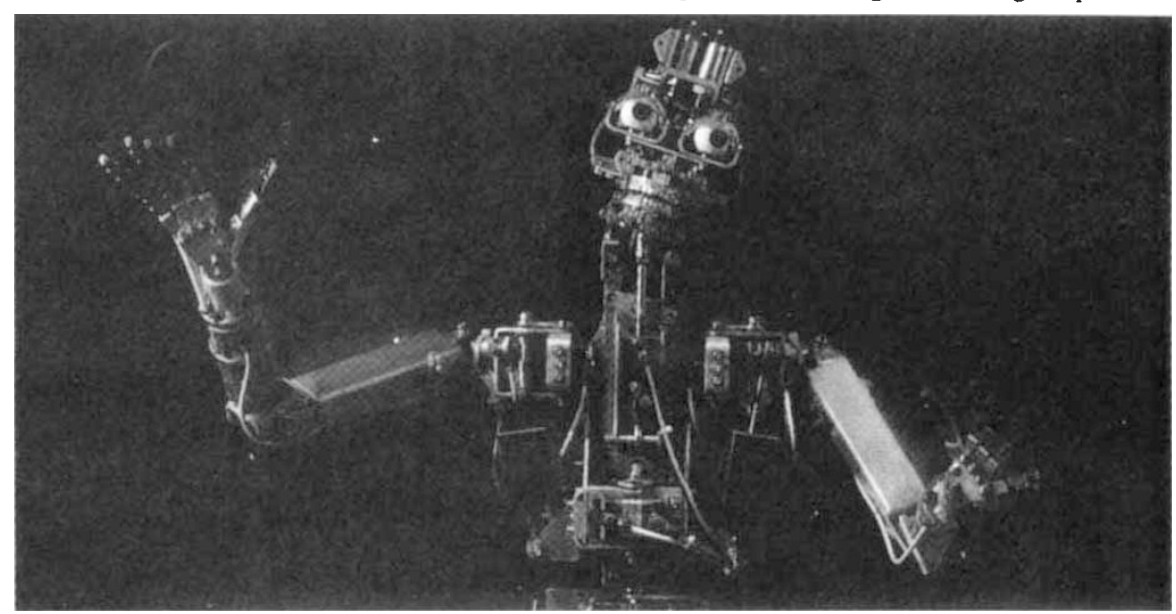

Robot Joral - a repulsive imitation of man? Photo: Len Jones.

treated to potted versions of the thoughts of philosophers from Plato to Wittgenstein. This is supplemented with essays by modern AI "philosophers" among whom are Daniel Dennet (". . . preoccupation with the Turing test is regrettable . . ."), Sherry Turkle (". . . [have] people always thought like machines? ...") and Douglas Hofstadter ("...I am an unabashed pusher of the Turing test. . ."). The essays are more interesting than the potted philosophy, with something in them both for those who know the arguments and those who do not.

It is hard to separate the mathematical basis for artificial intelligence from that of computer science in general. But this is attempted in a short chapter which is largely on logic. Kurzweil is much impressed by the generality of the "NOR" logical function and the fact that any digital machine could be constructed out of a sea of NOR logical "gates". This is fine, but displays a curious gap in what is otherwise quite a thorough coverage. There is no reference to the pioneering work of John von Neumann on the general logical theory of automata and self-reproduction in cellular automata. These make for a far more interesting way of approaching the "universality" problem than through the NOR gate. Mathematical roots are followed by technological roots, and it is in this area that the book comes to life. The history of both the mechanical side and the electronic side of computers (rather than AI) is well illustrated and appealingly written.

The second half of the book concentrates on the more recent aspects of $\mathrm{AI}-$ knowledge-based systems. Within this, Kurzweil's appreciation of the problems of recognizing visual and auditory patterns leads to lucid descriptions of some simple ways of solving these. After all, such methods have led to the success of his own machines. But such is the snobbery of researchers in rule-based systems, that I doubt they would accept hard-nosed programming methods such as "template matching" as being important

to AI. Also, those who are looking for a description of current debate in neural networks will find only a passing reference to the subject. This may be ironic as, not only does this field provide some hope of achieving some unaccomplished targets in AI, but also puts a bomb under the philosophical debate, centred as it is on the relevance of needing to represent intelligence by a set of computable rules. Neural systems, like living systems, learn and adapt, leading to an emergent intelligence within the machine rather than one that is entirely the responsibility of a programmer

Despite the limitations, this is an adventurous book. Both novices and experts will find in it something new and educational. For me, for example, the high spot was the discovery of Margaret Litvin's essay, which does more to prove the supremacy of the wisdom of people than anything a computer is ever likely to accomplish. To those who argue that some day computers will act as if they understand, think, love and hate, Margaret says: "Why can't scientists enjoy people, who really do these things?". Is the AI dilemma a result of scientific envy of naturally produced intelligent beings, who do things so well?

Igor Aleksander is in the Department of Electrical Engineering, Imperial College of Science, Technology and Medicine, Exhibition Road, London SW7 2BT, UK. who enjoys playing God. 